\title{
Selective Detection of Gaseous Ammonia with Specifically Functionalized Silicon Photonic Microring Resonator: Towards Low Cost and Portable Breath Monitoring
}

\author{
Nebiyu A. Yebo ${ }^{1 *}$, Sreeprasanth Pulinthanathu Sree ${ }^{4}$, Elisabeth Levrau ${ }^{3}$, Christophe Detavernier ${ }^{3}$, Zeger \\ Hens $^{2}$, Johan A. Martens ${ }^{4}$, Roel Baets ${ }^{1}$ \\ ${ }^{I}$ Ghent University-IMEC, Photonics Research group, INTEC, Sint-Pietersnieuwstraat 41, 9000 Gent, Belgium \\ ${ }^{2}$ Ghent University, Physics and chemistry of nanostructures, Krijgslaan 281-S3, 9000 Gent, Belgium \\ ${ }^{3}$ Ghent University, Dept. of Solid State Sciences, Krijgslaan 281/S1,Gent B-9000, Belgium \\ ${ }^{4}$ KU Leuven, Centre for Surface Chemistry and Catalysis, Kasteelpark Arenberg 23, Leuven B-3001, Belgium \\ nayebo@intec.ugent.be
}

\begin{abstract}
We demonstrate a selective ammonia sensor based on a silicon photonic microring resonator functionalized with nanoporous silicate films. The potential of this sensor as a low cost, portable and real-time medical breath monitoring device is discussed.

OCIS codes: (130.0130) Integrated Optics; (130.6010) Sensors
\end{abstract}

\section{Introduction}

In the past five years, silicon photonics for on-chip biomedical and environmental sensing has gained an increasing research interest [1-4]. A growing need in mass fabricated detection tools in the health care,

environmental and industrial sectors has been the major drive for silicon photonic sensors. With this regard, various integrated photonic device architectures are being explored to address important sensing features such as simplicity, multiplexing and device footprint [1-4]. A number of capabilities make the silicon photonics platform attractive for challenging sensing applications. Dense sensor integration and high refractive index sensitivity are among these features. Straightforward multiplexing of sensors also means that parallel detection of multiple analytes is readily achievable on a single chip. This opens opportunities for lab-on-chip applications whereby significantly minimizing sample volumes and the cost of laboratory processes as compared to traditional tools.

Apart from biomolecule detection in the liquid phase, silicon photonic sensors for a range of gas sensing applications are being explored recently [2]. Some of the areas integrated gas sensors can have a major impact are: point-of-care diagnostics in the health care sector, control and monitoring of environmental pollution, energy efficient and real-time process control in industrial applications, and threat detection. The bio-medical sensing market, in particular, is expected to rise due to an increasingly aging population, more spending in the heath care, and a growing shift towards point-of-care diagnostics. The detection of exhaled gaseous compounds is expected to have a key role in patient diagnosis and continual health monitoring. A human breath consists over 400 volatile compounds of which some have been recognized as biological indicators to specific health conditions. Nitric oxide (NO), ammonia (NH3), and acetone are some of these compounds indicated to have correlations with medical cases such as asthma, renal failure and oral diseases. For instance, a high correlation between blood urea nitrogen (BUN) and breath ammonia level in kidney patients has been reported [5]. The BUN test is currently the standard technique for monitoring kidney patients under dialysis treatment. However, this technique involves an invasive and lengthy laboratory procedure. As a consequence, there has been a growing interest for breath ammonia detection as a fast, real time and non-invasive alternative [5].

Various gas detection technologies exist today. However, traditional bulk gas sensors such as electrochemical sensors, gas chromatographs, mass spectrometers, and spectrophotometers, typically suffer from large size, complexity and high cost. Moreover, low cost technologies such as metal oxide semiconductor (MOS) sensors are often characterized by lack of specificity. Mass produced chip-scale silicon photonic gas sensors meeting application specific measures such as sensitivity and specificity can immensely enjoy cost, size and energy related befits over the traditional technologies, hence, promising a rapid contribution to the sensing market.

In this paper, we report a selective detection of gaseous ammonia using a silicon photonic microring resonator (MRR) functionalized with acidic nanoporous aluminosilicate films. The early stage $\mathrm{NH}_{3}$ sensor presented here shows fast, reversible, and selective response with respect to an important interfering gas, $\mathrm{CO}_{2}$. Two alternative approaches are used to functionalize the silicon MRRs with $\mathrm{NH}_{3}$ selective films. In the first route, an aluminosilicate microporous film, inspired by the synthesis of zeolites, was applied. In the second approach, a mesoporous silica film is deposited and functionalized with aluminum using Atomic Layer Deposition (ALD). The capability to specifically and rapidly detect gaseous compounds on optical chips, as demonstrated here, sheds a light 
on the future of low cost and highly portable silicon photonic sensors for complex gas analysis in various challenging applications
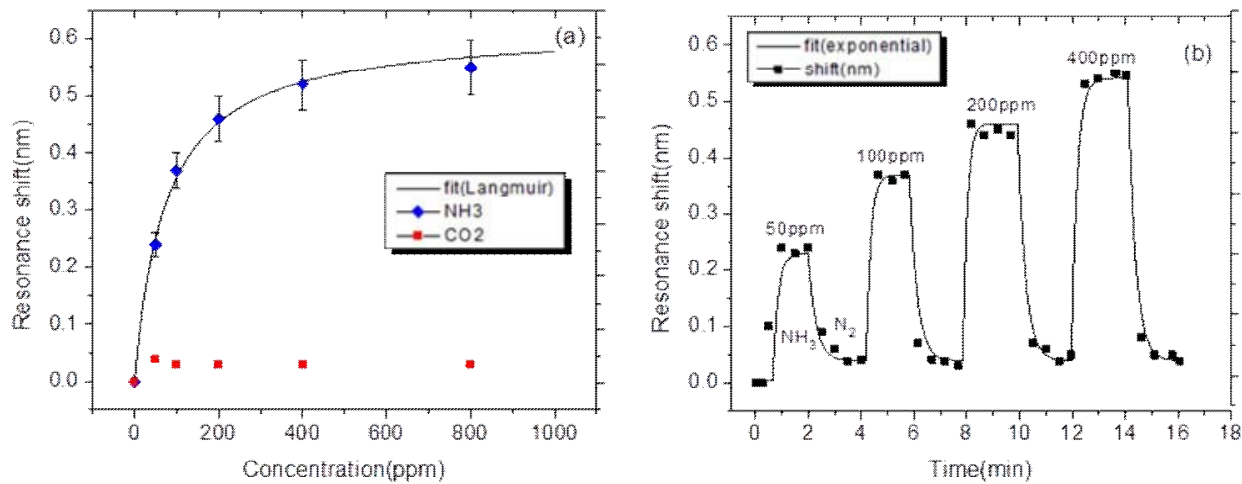

Figure 1 Response from a microporous acidic film coated MRR (a) resonance shift with respect to $\mathrm{NH}_{3}$ and $\mathrm{CO}_{2}$ concentrations (b) response and recovery with time at $\mathrm{NH}_{3}$ concentrations of 50,100,200 and $400 \mathrm{ppm}$ introduced for two minutes.

The resonance shifts of the MRR with microporous aluminosilicate coating upon exposure to different ammonia concentrations are shown in figure 1 (a). A distinct sensitivity to $\mathrm{NH}_{3}$ over $\mathrm{CO}_{2}$ is observed in $0-800 \mathrm{ppm}$ concentration range. The response to $\mathrm{NH}_{3}$ fits well $\left(\mathrm{R}^{2}=0.9946\right)$ to Langmuir adsorption isotherm with a linear response extending to $200 \mathrm{ppm}$ [2]. The Langmuir equilibrium constant and the maximum wavelength shift calculated with this fit are ca. $13.5 \times 10^{3}$, and ca. $620 \mathrm{pm}$, respectively. The response to $\mathrm{CO}_{2}$ is low and saturates at only ca. $30 \mathrm{pm}$. The error bars represent 7-10\% standard deviation observed over three measurements taken on the same sample. Interestingly, at a given $\mathrm{NH}_{3}$ concentration, an equilibrium response is reached in less than 30 seconds while over $95 \%$ recovery is reached within 60 - 90 seconds. The response and recovery of the $\mathrm{NH}_{3}$ sensor with respect to time fitted to exponentials is shown in figure 1 (b). A slight change of the base signal was observed after the first exposure while the responses to later $\mathrm{NH}_{3}$ exposures almost fully recovered.

A detection limit below $5 \mathrm{ppm} \mathrm{NH}_{3}$ is estimated for this early stage sensor. Our recent result have shown that significant improvement in the detection limit is readily achievable by tailoring the surface properties of the nanpororous $\mathrm{NH}_{3}$ sensitive silicate films. Such a selective sensor in a $\mathrm{CO}_{2}$ rich environment paves a ways towards a highly portable and low cost point-of-care device for breath $\mathrm{NH}_{3}$ detection.

\section{References}

[1] De Vos, T. Claes, Y. D. Koninck, S. Popelka, E. Schacht, R. Baets, and P. Bienstman, "Multiplexed antibody detection with an array of silicon-on-insulator microring resonators,” IEEE Photonics Journal 9, 225-235 (2009).

[2] N. A. Yebo, P. Lommens, Z. Hens and R. Baets, "An integrated optic ethanol vapor sensor based on a silicon-on-insulator microring resonator coated with a porous $\mathrm{ZnO}$ film," Optics Express, 18, 11859-11866 (2010).

[3] A. Ramachandran, S. Wang, J. Clarke, S. J. Ja, D. Goad, L.Wald, E. M. Flood, E. knobbe, J.V. Hryniewicz, S. T. Chu, D. Gill, W. Chen, O. King and B.E. Little, "A universal biosensing platform based on optical micro-ring resonators," Biosensors and Bioelectronics 23, 939-944 (2007).

[4] M. Iqbal, M.A. Gleeson, B. Spaugh, F. Tybor, W.G. Gunn, M. Hochberg, T. Baehr-Jones, R.C. Bailey and L.C. Gunn, "Label-Free Biosensor Arrays Based on Silicon Ring Resonators and High-Speed Optical Scanning Instrumentation,” IEEE Journal of Selected Topics in Quantum Electronics 16,654-661 (2010).

[5] L. R. Narasimhan, W. Goodman and C. K. N. Patel, "Correlation of breath ammonia with blood urea nitrogen and creatinine during hemodialyis," PNAS 98, 4617-4621 (2001). 